\title{
Digital Evidence Identification on Google Drive in Android Device Using NIST Mobile Forensic Method
}

\author{
Anton Yudhana ${ }^{1}$, Rusydi Umar ${ }^{2}$, Ahwan Ahmadi ${ }^{3}$ \\ ${ }^{1}$ Department of Electrical Engineering, Universitas Ahmad Dahlan (UAD) \\ 2,3 Department of Informatics Engineering, Universitas Ahmad Dahlan (UAD) \\ Yogyakarta, Indonesia \\ 1eyudhana@ee.uad.ac.id, ${ }^{1}$ rusydi_umar@rocketmail.com, ${ }^{3}$ ahwanahmadi71@gmail.com
}

\begin{abstract}
The use of cloud storage media is very popular nowadays, especially with the Google Drive cloud storage media on smartphones. The increasing number of users of google drive storage media does not rule out the possibility of being used as a medium for storing illegal data, such as places to store negative content and so on. On a smartphone with an Android operating system that has a Google Drive application installed, digital evidence can be extracted by acquiring and analyzing the system files. This study implemented a mobile forensic method based on guidelines issued by the National Institute of Standards of Technology (NIST). The results of this study are presented in the form of data recovery in the deleted Google Drive storage media, which results in the form of headers of the data type in the form of deleting account names, deleted file types, and timestamp of deleted files. Digital evidence obtained with 59 Axiom Magnet software found in the Entry227 file, with 46 files, 8 image files, video 3, zip 2, rar 4, pdf, 20, docx 4, pptx 2, Application 1, Database 2 and 15 files are only folder names that do not have data.
\end{abstract}

Keywords: Google Drive, NIST, Forensics, Mobile

\section{INTRODUCTION}

The use of storage media is currently very developed because more and more data is circulating and the inability of a storage medium or storage to store data, then a cloud storage storage media is created. This technology has significant potential to reduce costs and efficiency in storage [1]. Cloud storage is one of the remote data storage techniques that are interconnected with personal computers when connected to the internet [2]. Storing data online using cloud storage service media is one solution for storing data [3], [4]. Storage media applications offered by smartphones such as practicing bring and are supported by physical and cloud storage media [5]. Cloud storage services can offer greater storage flexibility and availability, with almost unlimited storage space, as well as the ability to synchronize data between multiple devices [6], [7]. The benefits of using cloud storage services are interesting, but security and privacy concerns are a major concern in cloud services [8]. The use of cloud storage media is increasing, it 
does not rule out the possibility of abuse of cloud storage storage media as an illegal data storage medium. Illegal data, such as videos, immoral images, pirated applications, fake documents. Illegal data that has been deleted on cloud storage can be used as digital evidence of cases of violations of the ITE Law. Digital evidence is expected to be another alternative to uncover a digital crime [9], [10], [11]. In uncovering cases of digital crime, computer science and technology are needed for the analysis and examination of digital evidence known as digital forensics [12], [13]. Digital evidence can be used as a law enforcement tool in handling digital cases obtained from cloud storage media on smartphones [14]. Digital forensics allows an analysis to recover a fact or event that is hidden in nature [15]. Digital forensics has two models, traditional forensic and live forensic. This study applies traditional forensic methods on Google Drive on an Android smartphone. The services provided by users get $15 \mathrm{~GB}$ of free storage space, including word processing, spreadsheet applications, and presentations, even program files with exe extensions. The $15 \mathrm{~GB}$ of storage space must be shared with a Gmail account, photos uploaded to Google+, and every document created on Google Drive [16].

\section{METHODS}

Digital forensic aims to analyze and reconstruct an event related to a computer or digital artifact [17]. Digital forensic methods and processes have been developed by forensic investigators and practitioners [18]. This study applies a method for the National Institute of Standards and Technology (NIST) which is a forensic method for analyzing digital evidence on a smartphone media [19]. The flow of the NIST method as shown in Figure 1.
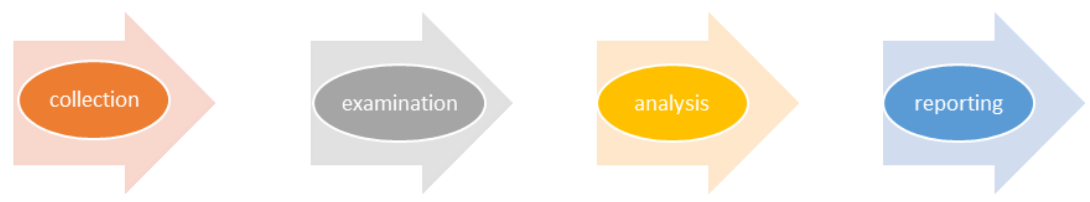

Figure 1. The NIST method for mobile forensic stages

The flow must be done in a mobile forensic process, these stages include:

\subsection{Collection/Preservation}

This stage is also called the preservation stage. This stage is the process of collecting, identifying, labeling, recording, and retrieving evidence in the form of hardware which data will be taken to be used as digital evidence of a digital crime case. This process is carried out by following data integrity safeguard procedures. Data integrity can be maintained by isolating physical evidence and making backups in the form of cloning or image files from physical evidence. Figure 2 shows the flow of the Collection stage. 


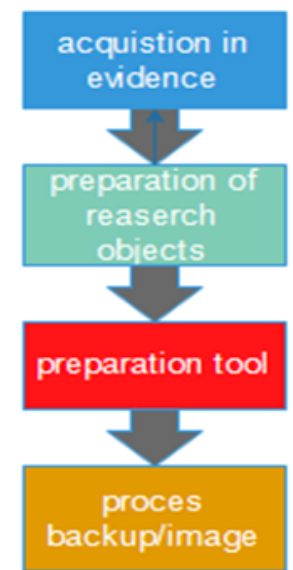

Figure 2. Collection process

\subsection{Examination}

Processing data collected digitally forensics using a combination of various scenarios, both automatically and manually, as well as assessing and issuing data according to needs while maintaining data integrity. Figure 3 describes the stages of the Examination process.

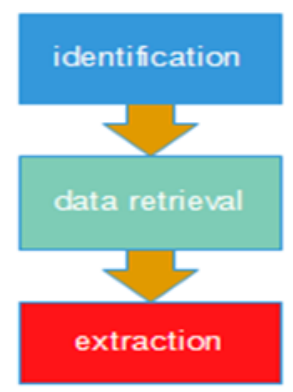

Figure 3. Examination process

\subsection{Analysis}

The process of analysis is carried out on the results of the examination with methods that have been justified technically and legally to obtain useful information and answer questions that are a reference or as a driver in conducting collection and examination. Figure 4 describes the flow of the analysis phase. Figure 6 describes the stages of the Analysis process. 
Figure 4. Process analysis

\subsection{Reporting}

The final result of an analysis process is a report. Reports from an analysis can be in the form of written reports needed as reports for documentation or oral reports in the form of presentations. The reporting process is explained in a groove like Figure 5.

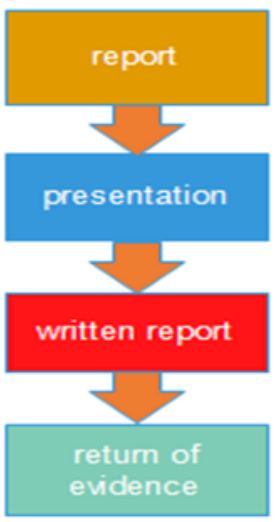

Figure 5. Reporting Process

\subsection{Research Tools and Materials}

There are 2 types of tools used in this study, namely tools in the form of hardware and software devices. The hardware used in this study is in the form of one smartphone device that is used as a test material, a computer as a workstation for forensic analysis and a USB Connector as a connecting medium between smartphone and workstation devices. 
Table 1. Hardware requirements

\begin{tabular}{cccc}
\hline No & Device name & Specification & Description \\
\hline 1 & Notebook & $\begin{array}{c}\text { AMD A10-9600P RADEON R5 } \\
\text { HP, Windows 10 64 Bit. }\end{array}$ & Workstation \\
& & Samsung Galaxy V Plus, OS & Hardware (test material) \\
3 & Smartphone & Android 4.4 KitKat & Media is connecting smartphone \\
with workstations
\end{tabular}

Table 2. Software requirements

\begin{tabular}{ccc}
\hline No & Device name & Description \\
\hline 1 & MOBILedit Forensic & Express \\
2 & Magnet Axiom & Forensic tool for imaging \\
3 & Forensic Connector & Media is connecting forensics tool \\
4 & Google Drive Smartphone & Ondroid
\end{tabular}

\section{RESULT AND DISCUSSION}

This research is an effort in analyzing evidence by applying the mobile forensic method on an Android smartphone by utilizing a forensic tool that will be tested for its performance, on a smartphone also already installed a google drive application. To analyze the evidence, a crime scenario is created in which a smartphone user saves a drug photo that will be circulated. The photo is saved on the smartphone Google drive. From the example of this scenario, it is assumed that the smartphone has been secured by the officer. Then the Investigation Team followed up on the smartphone found by making a copy of the system from the device so that the authenticity was maintained, and analyzed the evidence contained in the google drive application.

\subsection{Collection/Preservation}

The preservation stage is the first stage to secure evidence found by investigators or investigators. In this case, the investigator collected evidence from the owner, the evidence obtained in the form of 1 cellphone Samsung Galaxy V Plus with specifications OS, Android 4.4 KitKat, 6GB RAM, and inside it installed the Google Drive application. To avoid changing data on the smartphone the isolation process is needed by activating the Airplane mode feature. This feature is enabled to stop all data connectivity that can change the data integrity in the smartphone. This process is carried out with the aim of maintaining data integrity. Data integrity protection is done by physical proof isolation techniques and backup data with image files from the smartphone physical evidence using the MOBILedit FORENSIC EXPRESS tool. Examination of the google drive 
application that has been installed on the smartphone is done by acquiring data when the smartphone is on. To obtain digital evidence that you want to identify in the outlined case scenario, which is contained in the google drive application. The application used in this study is Magnet Axiom, which is one of the forensic tools that can be implemented for smartphones supported by data cables as a link between smartphones and Forensic Magnet software and then acquisitions.

\subsection{Examination}

The next stage is the process of retrieving data from the results of the MOBILedit FORENSIC EXPRESS image tool, then the image file data is extracted with the Axiom Magnet tool. After carrying out the extraction process, the inspection of the location of the evidence was located. Based on the results of the examination of the extraction results obtained 24 file partitions, where one of the partition files contained the location of the google drive file sought. Information from the partition location and google drive data is explained in Table 3.

Table 3. detailed information about the location of the google drive data partition

\begin{tabular}{ccc}
\hline No & Location of the google drive data partition & Information \\
\hline 1 & Partition 24 (EXT-family, 2.26 GB & $\begin{array}{c}\text { Shows the number of system partitions on } \\
\text { the smartphone }\end{array}$ \\
2 & data & $\begin{array}{c}\text { Shows file system data in partition 24 (EXT- } \\
\text { Family) }\end{array}$ \\
3 & Com.google.android.apps.docs & $\begin{array}{c}\text { Shows the location of the google drive folder } \\
\text { from data partition 24 (EXT-Family) }\end{array}$ \\
\hline
\end{tabular}

\subsection{Analysis}

The Google drive system is in the can. google. android. apps. docs folder in the file that allows the data the investigation team is looking for. The google drive file system can be explained in Table 4

Table 4. Description data from the google drive file system

\begin{tabular}{ccc}
\hline No & Data from the google drive file system & Information \\
\hline 1 & com.google.android.apps.docs & Location of the Google Drive data system \\
2 & evidence & Fill in the data on the Google Drive system \\
folder
\end{tabular}

From the process of analyzing Partition 24 system files (EXT-Family, 2.26 GB) that refers to the com. google. android. apps. docs folder, there is a database system, where the database contains information about what files are on Google actor's drive. Table 5 shows the analysis of the Partition 24 system files (EXTFamily, 2.26 GB). 
Table 5. description Indication of evidence

\begin{tabular}{ccc}
\hline No & Indication of evidence & Information \\
\hline 1 & Com.google.android.apps.docs & Location of Google Drive data \\
2 & DocList.db & Google Drive data base system \\
3 & Entry2227 & Location of all types of files on \\
& & Google Drive \\
\hline
\end{tabular}

The process analysis of the Entry 227 data is shown in Table 6 by analyzing erased data.

Table 6. Data entry 227

\begin{tabular}{ccc}
\hline No & Name & Information \\
\hline 1 & Entry_id & 59 \\
2 & Title & Heroin.jpg \\
3 & Owner & Ahwanahmadi71@gmail.com \\
4 & OwnerPictureUrl & https://h6.googleusercontent.com/-ZilZwNF- \\
bC8/xxxxxxxxxx \\
5 & creationTime & 1539103369317 \\
6 & lastModifiedTime & 1539103369317 \\
7 & lastModifierAccountName & Ahwan Ahmadi \\
8 & lastOpenedTime & 15401057456667 \\
9 & recencyTime & $15401057456 x x x$ \\
10 & ModifiedByMeTime & 1539103369317 \\
11 & mimeType & Image/jpeg \\
12 & trashed & 3 \\
\hline
\end{tabular}

Table 7 shows the results of the data analysis deleted from the google drive application which is in the Entry 227 data.

Table 7. Description of Table 6

\begin{tabular}{ccc}
\hline No & Name & information \\
\hline 1 & Entry_id & Number of files on Google Drive \\
2 & Title & file name \\
3 & Owner & File owner \\
4 & OwnerPictureUrl & Image of owner from Url \\
5 & creationTime & When the file was created \\
6 & lastModifiedTime & The last time was modified the file \\
7 & lastModifierAccountName & The last account name that changed the file \\
8 & lastOpennedTime & The last time the file was opened \\
9 & recencyTime & The time period for opening the file \\
10 & modifiedByMeTime & When the file is changed \\
11 & mimeType & File type \\
12 & trashed & Deleted \\
\hline
\end{tabular}




\subsection{Reporting}

At this stage the results of digital evidence that has been acquired and has undergone an analysis process carried out by the investigation team are then reported as the results of the findings of the analysis process. Table of process results Analysis of the Axiom Magnet tool is shown in Table 8.

Table 8. Results of analysis of the Axiom Magnet tool

\begin{tabular}{cccc}
\hline No & Type File & Number of File types & Deleted type of file type \\
\hline 1 & Image & 8 & $\checkmark$ \\
2 & Video & 3 & $\checkmark$ \\
3 & Zip & 2 & - \\
4 & Rar & 20 & - \\
5 & PDF & 4 & - \\
6 & Docx & 2 & $\checkmark$ \\
7 & Pptx & 1 & - \\
8 & Aplication & 2 & $\checkmark$ \\
9 & Data Base & & - \\
\hline
\end{tabular}

The stages that have been carried out in the sample case scenario can also be applied in other cybercrime cases. Complexity in finding and obtaining digital evidence for cybercrime cases using smartphone media such as those mentioned in the report mentioned by RSA in 2013The stages that have been carried out in the sample case scenario can also be applied in other cybercrime cases. Complexity in finding and obtaining digital evidence for cybercrime cases using smartphone media such as those mentioned in the report mentioned by RSA in 2013 [20]. the availability of forensic tools is also very supportive to overcome the complexity that may be faced by digital investigation teams in dealing with digital crime. This research is at least a reference in conducting further studies with the latest cases such as social media and cloud storage media on smartphones.

\section{CONCLUSION}

The results of the analysis and discussion that have been made and explained in this study, there are a number of things that can be concluded including: the NIST method in digital forensic processes can be applied to cloud storage media case studies using the help of Axiom Magnet software. From the data obtained with the Axiom Magnet software, 59 files were found in the Entry227 file, with 46 files, 8 image files, video 3, zip 2, rar 4, pdf, 20, docx 4, pptx 2, Application 1, Database 2 and 15 files are only folder names that do not have data. Based on the scenario made by the investigator to find the image file sought, it is in the image type file where of the 8 image files found 2 of them have been deleted, including the files searched for, 3 video files deleted there are 2 files, application files also deleted and files docs whose number 4 is found 1 deleted file that is known to be based on its trashed value. From the results of testing with the Magnet Axiom 
software the file was found, but the researcher could not open the file, the researcher could only find out the file name and type. From the description of the results of the above research, the researchers also suggested the further development by using more detailed and complete forensic methods and trying out mobile forensic tools that could be used to find evidence on cloud storage media. The use of methods and supported by other forensic tools will provide more satisfying results by staying referral to mobile forensic standards and with more in-depth analysis also included with more complete report results.

\section{REFERENCES}

[1] Armbrust, M., Fox, G., Griffith, R., Joseph, A., \& RH. (2009). Above the clouds: A Berkeley view of cloud computing. Univ. California, Berkeley, Tech. Rep. $U C B, 07-013$.

[2] Easwaramoorthy, S., Thamburasa, S., Samy, G., Bhushan, S.B., \& Aravind, K. (2016). Digital Forensic Evidence Collection of Cloud Storage Data for Investigation. International Conference On Recent Trends In Information Technology, 1-6.

[3] Mager, T., Biersack, E., \& Michiardi, P. (2012). A measurement study of the Wuala on-line storage service. 2012 IEEE 12th Int. Conf. Peer-to-Peer Comput. P2P 2012.

[4] Faheem, M., Le-Khac, N.A., \& Kechadi, T. (2017). Toward a new mobile cloud forensic framework. 2016 6th Int. Conf. Innov. Comput. Technol. INTECH 2016.

[5] Riadi, I., Umar, R., \& Firdonsyah, A. (2018). Forensic tools performance analysis on android-based blackberry messenger using NIST measurements. Int. J. Electr. Comput. Eng., 8(5), 3991-4003.

[6] Mulazzani, M., Schrittwieser, S., Weippl, E., Leithner, M., \& Huber, M. (2011). Dark Clouds on the Horizon: Using Cloud Storage as Attack Vector and Online Slack Space. USENIX Secur. 8, pp. 11.

[7] Martini B. \& Choo, K.K.R. (2013). Cloud storage forensics: OwnCloud as a case study. Digit. Investig., 10(4), 287-299.

[8] Subashini, S. \& Kavitha, V. (2011). A survey on security issues in service delivery models of cloud computing. J. Netw. Comput. Appl., 34(1), 1-11.

[9] Yudhana, A., Riadi, I., \& Anshori, I. (2018). Analisis Bukti Digital Facebook Messenger Menggunakan Metode Nist. IT J. Res. Dev., 3(1), 13-21.

[10] Anwar, N., \& Riadi, I. (2017). Analisis Investigasi Forensik WhatsApp Messanger Smartphone Terhadap WhatsApp Berbasis Web. J. Ilm. Tek. Elektro Komput. dan Inform., 3(1), 1-10.

[11] Putra, R.A., Fadlil, A., \& Riadi, I. (2017). Forensik Mobile Pada Smartwach Berbasis Android. Jurti, 1(1), 41-47.

[12] Umar, R., Riadi, I., \& Muthohirin, B.F. (2018). Acquisition Of Email Service Based Android. KINETIK, 3(4), 263-270.

[13] Faiz, M.N., Umar, R., Yudhana, A., \& Dahlan, U.A. (2016). Analisis Live Forensics Untuk Perbandingan Kemananan Email Pada Sistem Operasi Proprietary. J. Ilm. Ilk., 8(3), 242-247.

[14] Umar, R., Riadi, I., \& Maulana, G. (2017). A Comparative Study of Forensic Tools for WhatsApp Analysis using NIST Measurements. Int. J. Adv. Comput. Sci. Appl., 8(12), 69-75.

[15] Fadlil, A., Riadi, I., Aji, S., \& Dahlan, U.A. (2017). Pengembangan sistem pengaman jaringan komputer berdasarkan analisis forensik jaringan. J. Ilmu Tek. Elektro Komput. dan Inform, 3(1), 11. 
[16] Juliandi, A. (2014). Personal Storage. pp. 0-14.

[17] Forensics, D. (2009). Hashing and Data Fingerprinting in Digital Forensics.

[18] Faiz, M.N., Prabowo, W.A., \& Sidiq, M.F. (2018). Studi Komparasi Investigasi Digital Forensik pada Tindak Kriminal. J. Informatics, Inf. Syst. Softw. Eng. Appl, 1(1), 63-70.

[19] Kent, K., Chevalier, S., Grance, T., \& Dang, H. (2006). Guide to integrating forensic techniques into incident response.

[20] EMC. (2014). The Current Trend in Cybercrime 2014 - An Inside Look at the Changing Threat Landscape. The Current State Of Cybercrime 2013, October 2013, pp. 1-9. 